\title{
Designing with Only Four People in Mind? - A Case Study of Using Personas to Redesign a Work-Integrated Learning Support System
}

\author{
Amir Dotan, Neil Maiden, Valentina Lichtner, and Lola Germanovich \\ Centre for Human-Computer Interaction Design, City University London \\ Northampton Square, London, EC1V OHB
}

\begin{abstract}
In this paper we describe and reflect on the use of personas to redesign the $3^{\text {rd }}$ prototype of APOSDLE - a system to support informal learning and knowledge transfer in the workplace. Based on the results of a formative evaluation of the $2^{\text {nd }}$ prototype we used personas to explore how users' goals, behaviour and preferences could be communicated to project members during a two-day design workshop, in order to ensure useful and usable design solutions. We actively involved stakeholders representing the target market throughout the process as they helped to create, validate and interpret the four personas we used. By doing so we aimed to address methodological weaknesses and practical limitations of using personas, primarily those relating to the validity of the personas used and the way they are interpreted. We reflected on how effective the personas were by referring to data generated during the workshop and discussion transcripts. As reported by others, and as we have experienced ourselves, using personas can be quite challenging as rich narrative descriptions are expected to produce insight and design solutions. In light of this challenge, we contribute a case study illustrating how personas were implemented in a real world situation to engage project members with user information and drive the design process. We specifically discussed the strengths and weaknesses of actively involving stakeholders in creating and using personas.
\end{abstract}

Keywords: Personas, User-Centred Design, Work-Integrated Systems, Interaction Design, Usability.

\section{Introduction}

APOSDLE (Advanced Process-Oriented Self-Directed Learning Environment) is a 48 month research and development project involving twelve European organisations from five countries. It aims to support work-integrated learning by providing people working in knowledge-intensive industries the infrastructure needed to acquire, understand and communicate knowledge. The system detects and analyses the user's computer activity in order to infer a task the user is working on and suggest relevant resources that might prove helpful. These resources are tailored to the user's 'work context' and profile information, which includes data such as inferred level of knowledge based on previous interactions with the system among other parameters. Following a formative evaluation of the $2^{\text {nd }}$ system prototype, which highlighted 
various usability problems, it was decided to introduce personas in order to explore how user information could be integrated more effectively to inform the design of the $3^{\text {rd }}$ and final prototype. Personas are a vivid 'day in the life' description of fictional individuals representing key user groups used to communicate their goals, behaviour and preferences in an engaging and practical way $[9,12]$. Establishing a shared understanding of key user groups was especially important ahead of a two-day design workshop involving 21 project members from different professional backgrounds. While the results of the formative evaluation served as the main catalyst for the redesign and provided valuable insight into users' experience using APOSDLE, they did not communicate the goals and work environment of those users in a memorable and actionable manner. Personas were regarded as a potential way to communicate such information to participants so it could be effectively referred to during the discussions.

In this paper we describe how we used personas and reflect on how effective it was to design with only a handful of specific individuals in mind. By doing so we provide a real life account that might inform others wishing to use the tool. While literature focuses on analysing field data to identify and establish personas, we chose to complement such data by collaborating with stakeholders in order to create and use the four personas. We discuss the strengths and weaknesses of this collaborative approach by presenting data gathered and observed during a two-day design workshop. We begin with an overview of the main research work carried out as part of the APOSDLE project before the personas were introduced. This is followed by a review of relevant literature. We then describe the methodology we implemented to create the personas. The results section includes an analysis of how the personas were used by referring to comments made by workshop participants and discussion transcripts. In the discussion section we address the strengths and weaknesses of our approach and personas in general as well as lesson learned from the experience.

\section{Previous Prototype Development Work}

The development of the APOSDLE project included a variety of research activities (e.g. creativity workshops, focus groups) executed in part to elicit requirements and evaluate the system. A functional prototype has been evaluated every year as part of an iterative development approach. Four of the twelve organisations involved in the project are companies seen as the target market of the APOSDLE system. Project members working in these four organisations (referred to in this paper as application partners) are familiar with and represent the future end users of the system (e.g. engineers, consultants). Their involvement proved important to ensure the project was aligned with the needs and work environment of its intended users. The need to consider additional design tools, which led to the use of personas described in this paper, arose following an extensive four-week formative evaluation of the $2^{\text {nd }}$ prototype [8]. Primarily work-based, it highlighted various usability problems ranging from ambiguous terminology to unclear system logic. A considerable number of users could not fully grasp how APOSDLE might benefit them at work [1]. We concluded the user interface had to be redesigned so it supported users' goals and knowledge needs more effectively. In order to achieve this objective we had communicate and remind 21 project members of those goals so they could be referred to during design 
discussions. The target audience of APOSDLE had been mapped from the outset; however the descriptions used lacked contextual data consisted of such abstract terms such as Knowledge Seeker and Knowledgeable Person. Such segmentation provided little if any useful data about the people the project was targeting and their work environment, therefore we decided to use personas instead.

\section{Previous Research into Personas}

Personas have become a popular tool since they were first introduced by Alan Cooper [3] as means of incorporating user information into the design process. By referring to carefully constructed rich descriptions of fictitious individuals, a design team aims to engage with 'real people' in order to assess how the product may or may not suit their goals. Similar to other techniques such as Cultural Probes [5], personas are meant to engage teams with useful information about end users, so that it can be referred to during the design process. This is often done in conjunction with other user-centred methods such as user testing as personas are not expected to provide all the answers regarding product usability and usefulness. Pruitt and Adlin [10] note the idea of designing for a very small set of concretely defined user archetypes is still fairly new and considered radical by many since the conventional mindset is to ignore the preferences of the individual user in order to satisfy those of a wider audience. However, Cooper [3] argues that contrary to common logic, it is better to design with a single person in mind rather than try to accommodate most people, assuming the single person being referred to is constructed correctly and is seen as a valid representation. Grudin and Pruit [6] list the following benefits of using personas: 1) They create a strong focus on users and work contexts 2) They utilise people's powerful ability to extrapolate from partial knowledge to create coherent wholes and project them into new settings 3) The act of creating personas makes assumptions about the target audience explicit 4) They provide a shared basis for communication 5) They focus attention on a specific target audience.

Using personas during the design process effectively creates numerous challenges, as they need to be memorable and engaging in order to have an effect $[9,11]$. People need to buy-into the personas and get to know them if they are expected to champion their goals. The personas also need to be used on a regular basis in order to influence the design process. Grudin and Pruit [11] reported on the successful use of personas at Microsoft; however they ended up developing different tools in order to communicate the personas and ensure they are believable representations. Similarly, Nieters et al. [9] also reported how using the tool required some degree of improvisation to make the personas memorable and engaging (the personas were eventually represented by plastic action figures). Such accounts demonstrate that in practice, personas are often used in different and at times creative ways, and are seen as flexible rather than a rigid tool. Chapman and Milham [2] present a sceptical view of personas and describe methodological weaknesses and practical limitations, which they argue undermine the validity of the tool. From a methodological perspective, they assert personas are difficult or impossible to verify and represent only a very small portion of the intended user population. In terms of practical limitation they point to the fact that it is not always clear how the personas are reconciled with other data and who is responsible 
for interpreting them. Existing literature about personas indicates the tool is debatable with various strengths and weaknesses. As we describe in later sections, we acknowledged methodological and practical weaknesses and made attempts to address them by collaborating with stakeholders to create the personas and involving them in the design process to support the interpretation of those personas.

\section{Methodology - Creating the Personas}

In this section we describe how the APOSDLE personas were created before being used during a two-day design workshop. We developed the personas based on empirical data gathered from observations and interviews as well as previous research carried out as part of the APOSDLE project (e.g. [6]). Given the four application partners' wealth of internal organisational knowledge, we decided to augment the standard persona creation process by getting them actively involved in creating and validating the personas. They were asked to produce a detailed description of employee types using a template we provided and based on their input, as well as additional data, we synthesised a set of four personas. By doing so we sought to address methodological and practical weaknesses expressed in the literature with regards to persona validity and the way the personas are interpreted and utilised. Apart from validating the personas the application partners were frequently consulted with during the design workshop when a persona was referred to. As we later describe, while this helped resolve ambiguity and disagreements it also contributed in our view to the personas being underused overall.

\subsection{Data Collection}

We provided the application partners with a template that included basic demographic details and sections frequently mentioned in literature about personas such as 'typical day at work', 'goals', 'dislikes' and 'attitude towards technology'. We received eight descriptions in total from three of the four application partners. A consultant and administrative assistant emerged as the main employee types in two consultancies. An engineering company provided four descriptions, which included a senior engineer and an intern. Based on these descriptions and empirical data we already had, we identified the following dimensions as the main distinctions between the different employee groups:

- Nature of work - Scientific and rigid (Engineering) versus creative and flexible (Consultancy). While the former relied heavily on a structured approach, calculations and precision, the latter required being able to adapt to clients.

- Level of experience - An experienced, possibly senior, employee versus a new employee, probably an intern.

While these dimensions were not new to us, and such user segmentation was identified early in the project, our goal was ensuring this data was made visible and engaging to all 21 participants during the workshop so it could help establish empathy with end users needs and circumstances. 


\subsection{Constructing the Personas}

We ended up constructing four personas based to a large extent on the ones provided by the application partners. The personas represented senior and junior employees in scientific and creative industries. The two primary personas represented senior employees in an engineering company (Pierre) and a senior consultant working in an innovation consultancy (Eva). One secondary persona represented an intern in an engineering company (Paul) and the other an administrative assistant in a consultancy (Lisa). Only one application partner listed an intern as a possible persona, therefore we considered this type of employees to be secondary compared to senior and experienced employees. We did not expect APOSDLE would equally satisfy all the personas given their different goals; hence the distinction between the primary and secondary personas i.e. the goals of a primary persona had priority over those of a secondary one. As can be seen from the following example, we produced a rich persona description that captured daily work routine as well as behavioural patterns that could inform the persona's goals, preferences and attitudes:

Eva -37 years old, married +1 and lives in Graz, Austria. She has PhD in Industrial Economics. She worked for two years as a junior consultant, mentored by a senior consultant before taking over projects. Her work requires her to be creative and constantly adapt to new situations created by working with new clients. She is often away from the office due to meetings or travels. She prefers as much face-toface communication with her clients. She is self-organised, self-directed and sometimes find herself overcommitted. She uses phone and email for contact colleagues, many meetings with other team leaders and heads of department. Eva uses mainly MS Office, two different web content management systems, two address databases, accounting system, Lotus Notes and internal Wiki. She is technologically interested, curious and open-minded. She is very busy and critical and therefore prefers to have a quick look in Google instead of searching her notebook or the server. Typical day: many meetings and emails, multitasking; deciding, planning, coordinating controlling and monitoring activities. In her free time she likes sports, she is an active triathlete. She is very interested in school topics and the education of her children and is chairman of the parents association at her childrens' school.

Each description included 10-15 related visuals that were meant to help participants extrapolate additional information by visualising various aspects of the written narrative. Using visuals to augment a persona's textual description is common practice [e.g. 9, 11], and similar to Gaver et al.'s [5] Cultural Probes techniques, is meant to generate further insight by engaging people with visual or tangible objects. In Eva's case the visuals included a mindmap, flipchart, a handshake, a train and Google's logo. They emphasised key attributes such as the creative and client-facing nature of her work, as well as the fact she often travels and is therefore out of the office. Before using the personas we asked the application partners to review and validate the personas we created by examining how well they represented employees in their organisation. We received positive feedback (see example below) and were satisfied the personas were a realistic representation and ready to be used.

I like very much the personas you have synthesized from the application partners' input. I really can recognize [our company's] two main groups of employees in Eva and Lisa. (Personal email correspondence)

\subsection{Introducing the Personas}

The personas were introduced at the beginning of the design workshop during an hour long 'familiarisation session'. 21 participants representing each project partner 
(programmers, learning experts, knowledge management scientists, application partners) took part in the workshop, which was facilitated by two of the authors. Since the participants were not familiar with personas the challenge we faced was two fold. First, they had to buy into the tool and appreciate how it could benefit the design process. Second, they had to accept the personas we developed as realistic representations of the end users. The familiarisation session was designed to introduce the personas and allow the participants to eventually perceive them as real individuals and advocate their goals. During the session each persona was projected on a screen one at a time and a facilitator introduced it and asked key questions in order to generate a discussion. The process had four main objectives:

1. Get participants to view the personas as real people they can relate to and empathise with;

2. Explore general assumptions about the personas and talk about their work and learning needs (explicit and implicit);

3. Explore specific assumptions regarding each persona's attitude towards the current version of APOSDLE e.g. what aspects of the system the persona would likely appreciate and find useful;

4. Explore similarities and differences between the four personas;

Each discussion began with the following key questions: "What kind of information is the persona likely to look for at work?" and "How does the persona find answers to questions she has at work?" These questions set the scene for a discussion about the personas' likely attitude towards the existing version of APOSDLE. The main questions we asked with regards to the system were: 'What aspects of the current APOSDLE prototype the persona would not like?' and 'What aspects of the current APOSDLE prototype persona would like?' Having the application partners present was useful as they contributed examples and helped resolve disagreements by either supporting or rejecting assumptions made by participants about the personas.

\section{Results - Utilising the Personas}

In this section we report how the personas were utilised during the workshop and the impact they have had on the redesign process. We first analyse the data gathered during the familiarisation session and then present a sample of transcripts from the discussions showing how the personas were used to inform various design decisions. In order to evaluate the impact the personas may or may not have had on the workshop we reviewed video footage of the two days and observed how the personas were used. We isolated the events where the personas were referred to in order to assess: 1) How much impact they had on the discussions 2) What was their impact in terms of redesign and 3) how the way they were utilised might have been affected by the participation of the application partners who helped create them.

\subsection{Familiarisation Session}

The familiarisation session resulted in general comments made by participants about the personas and assumptions as to how they were likely to perceive the existing version of APOSDLE. This part of the workshop lasted 63 minutes and generated a 
Table 1. Genereal comments and assumptions made about the personas

\begin{tabular}{|c|c|c|}
\hline & Rigid work process & Flexible work process \\
\hline 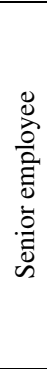 & $\begin{array}{l}\text { Pierre (Primary persona) } \\
\text { - } \text { Regarded as expert } \\
\text { - Responsible for training and supporting new } \\
\text { employees (interns) } \\
\text { - Set in his ways } \\
\text { - } \text { Contacts internal and external experts } \\
\text { - } \quad \text { Deaws who knows what in the company } \\
\text { - } \text { calculations } \\
\text { Would like to spread his knowledge and build } \\
\text { a positive reputation }\end{array}$ & $\begin{array}{l}\text { Eva (Primary persona) } \\
\text { - Knowledge needs depend on clients } \\
\text { - Frequently Out of the office } \\
\text { - Relies on past projects and peers' experience } \\
\text { - Deals with people (clients) } \\
\text { - Process influenced by clients }\end{array}$ \\
\hline 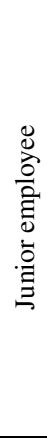 & $\begin{array}{l}\text { Paul (Secondary persona) } \\
\text { - } \quad \text { Question driven } \\
\text { - } \text { Internet savvy } \\
\text { - } \text { Willing to explore new things } \\
\text { - } \quad \text { Single task at a time } \\
\text { - Requires internal knowledge (procedures) } \\
\text { - Uses Google products } \\
\text { - } \quad \text { Does not know who to ask for help } \\
\text { - } \quad \text { Requires detailed and specific knowledge } \\
\text { - He has time to learn and likes to explore new } \\
\end{array}$ & $\begin{array}{l}\text { Lisa (Secondary persona) } \\
\text { - Supports others work } \\
\text { - Free to plan her work } \\
\text { - Work is influenced by requests made by } \\
\text { consultants } \\
\text { - Acts as a 'hub' in the office }\end{array}$ \\
\hline
\end{tabular}

lively discussion, which actively involved more than half of the participants. The personas were introduced and discussed in the following order: Pierre (22 minutes), Eva (16 minutes), Paul (13 minutes) and Lisa (11 minutes). Table 1 summarises the general points raised during the discussion about each persona; from personal preferences to the type of knowledge needs encountered at work.

The participants' comments enriched the personas' description and added important information. The application partners were referred to on a number of occasions to support or dismiss assumptions participants made, and their input was very useful. As can be seen, Pierre and Paul generate the highest number of comments. We can speculate on what were the reasons why the personas were not equally engaging: 1) Participants found it easier to relate to Paul and Pierre because they were more familiar with their work domain; 2) Pierre and Paul were seen as more relevant to the project; 3) The description and accompanying visuals associated with Pierre and Paul were clearer and possibly more engaging. A particular reason why Lisa was not as engaging as the other personas could be due to the fact she was the last persona to be introduced after more than 50 minutes. It is reasonable to assume that at thaint general fatigue set in and affected the degree of participation. In hindsight, it would have been better to discuss the personas in parallel and ensure they are each discussed for an equal amount of time. It is worth noting Lisa was included as a persona because two application partners described an assistant as a significant employee type. This did not automatically make her an end user of APOSDLE. However, we wished to explore ways APOSDLE could potentially benefit new user types. We believe the Lisa persona was also less engaging since it was not perceived as relevant to the 
project. We tend to exclude the possibility of gender-bias in this case since there was an equal number of men and women taking part in the discussions and overall in the workshop.

Table 2 summarises the comments expressed regarding each persona's likely attitude towards the $2^{\text {nd }}$ prototype. These issues were discussed following the general comments section while the participants were still engaged with a particular persona. As can be seen by the comments, participants felt comfortable inferring how the personas would likely perceive APOSDLE. This was encouraging and showed the first part of familiarisation session was effective as it enabled participants to extrapolate new information based on the persona description. Ensuring the discussion revolved around two questions i.e. what the personas would like and not like about APOSDLE, helped focus the responses and was very effective. It also helped ensure the comments would be comparable and could be easily grouped to uncover patterns,

Table 2. Comments and assumptions made regarding the personas' attitude towards APOSDLE

\begin{tabular}{|c|c|c|}
\hline & Rigid work process & Flexible work process \\
\hline 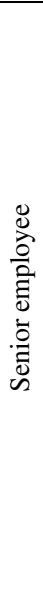 & $\begin{array}{l}\text { Pierre (Primary) } \\
\text { - Will not use task selection } \\
\text { - Will not use the Wiki to communicate } \\
\text { - Might not use APOSDLE's generated } \\
\text { Learning Events and jump straight to a PDF } \\
\text { without reading any introductory text } \\
\text { offered by the system } \\
\text { - Would appreciate collaboration options and a } \\
\text { place to discuss with others } \\
\text { - Would appreciate detailed and advanced } \\
\text { - } \text { - Wourch options } \\
\text { - Requires incentives to use the annotation tool } \\
\text { and 'tag' portions of resources according to } \\
\text { the domain }\end{array}$ & $\begin{array}{l}\text { Eva (Primary) } \\
\text { - Would consider APOSDLE as 'just another } \\
\text { tool' } \\
\text { - Would not use APOSDLE's process view } \\
\text { as her work is less about 'ticking boxes' } \\
\text { - Would be interested to get a quick overview } \\
\text { of her clients' work domain (especially if } \\
\text { she is not familiar with that work domain) } \\
\text { - Would likely compare APOSDLE to } \\
\text { Google and conclude it is not as good } \\
\text { - Would appreciate different work domains } \\
\text { to learn from about her clients } \\
\text { - Would appreciate collaboration options } \\
\text { within APOSDLE } \\
\text { Would appreciate being able to locate } \\
\text { experts using APOSDLE } \\
\text { Would consider APOSDLE a creative } \\
\text { concept }\end{array}$ \\
\hline 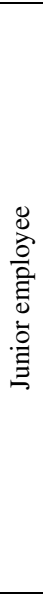 & $\begin{array}{l}\text { Paul (Secondary) } \\
\text { - Would not like the fact APOSDLE does not } \\
\text { interface with his favourite applications } \\
\text { - Would perceive APOSDLE as too difficult to } \\
\text { learn compared to web applications he uses } \\
\text { - Would not like the fact APOSDLE does not } \\
\text { run on Linux } \\
\text { - Would not understand the modelling termi- } \\
\text { nology used in APOSDLE } \\
\text { Would appreciate the fact that APOSDLE } \\
\text { highlights relevant portions of resources } \\
\text { instead of having to read through a 500 page } \\
\text { long PDF } \\
\text { - Would appreciate the fact that by effectively } \\
\text { using APOSDLE he does not need to ask } \\
\text { people questions all the time } \\
\text { He is not too concerned with privacy and } \\
\text { whether APOSDLE records his personal data }\end{array}$ & $\begin{array}{l}\text { Lisa (Secondary) } \\
\text { - Would not appreciate the lack of } \\
\text { interoperability with other collaboration } \\
\text { tools she uses at work } \\
\text { - Constantly changing her tasks could hinder } \\
\text { the automatic context detection process } \\
\text { - May not see APOSDLE as a tool that } \\
\text { directly supports her work }\end{array}$ \\
\hline
\end{tabular}


similarities and differences between the personas. The following assumptions focused participants' attention on key issues and served as the basis for the design session we describe in the next section.

The patterns which emerged during this discussion referred to mainly to APOSDLE's emphasis on structured learning support and how well it suited different personas. While experienced employees such as Eva and Pierre would likely appreciate various unique features of APOSDLE like the use of semantic models to link resources to tasks and topics, its focus on learning would probably deter them and appeal more to an inexperienced employee such as Paul. This had significant impact on functionality and terminology. Such assumptions were derived from the data presented in Table 1 as it was understood Paul had explicit learning needs and was question driven compared to Pierre who was more problem driven. Out of the four personas, Paul was seen as the only one that was given explicit learning tasks by his supervisors and was most likely to be in a 'training mode'. Once it was established that experienced employees like Eva and Pierre were less likely to require structured learning support, we began exploring how they might benefit from APOSDLE's various features in their everyday work. One significant outcome of this realisation was that the Search function became more prominent in the user interface; acknowledging it would be frequently sought by someone familiar with the work processes and who is interested in quick and easy access to information rather than following a step-bystep approach.

\subsection{Reviewing the $2^{\text {nd }}$ Prototype}

Following the familiarisation session, we presented participants the $2^{\text {nd }}$ prototype and began focusing on specific system components from the point of view of each persona. We focus on two of several examples, which illustrate how the personas were used to evaluate the prototype and inform design decisions. In the first case, participants were asked to evaluate APOSDLE's Learning Events concept. A Learning Event was an automatically generated collection of annotated documents, which included an introductory paragraph and a form allowing users to reflect and assess how well they have learned the material. A list of available Learning Events was displayed once the user selected a Learning Goal (e.g. "How to plan a creativity workshop") from a list of learning goals relating to a specific work task (e.g. "Run a creativity workshop"). The formative evaluation of the $2^{\text {nd }}$ prototype showed users struggled to make sense of Learning Events so assessing and redesigning this system component using the personas was important.

Facilitator A: Paul is probably the main recipient of Learning Events [according to the previously mentioned assumptions]. What Paul might want?

Participant 1(Knowledge management scientist): He had a Learning Goal. Otherwise he wouldn't end up with a Learning Event.

Facilitator A: Is Paul able to select his own Learning Goal? How did users react to Learning Goals in the formative evaluation?

Participant 2 (Application Partner A): They didn't understand what they meant.

Participant 3 (Application Partner B): Some of our users didn't understand the Learning Goal type (apply, understand etc.)

Participant 4 (Learning expert): Perhaps we should phrase them in a more intuitive manner. 


\begin{abstract}
Participant 5 (Programmer): The Learning Events were created to facilitate learning. I think we should reflect on the four personas and see if they have need for this information. We are discussing low-level details and terminology before addressing the users' real goals.

Facilitator B: There were two personas, Lisa and Eva, that we said need quick overview of material.

Facilitator A: What would Paul want to learn from?

Participant 4 (Learning Expert): he wants a definition

Participant 6 (Learning Expert): A slider could be used to show different level of learning support depending on the user's learning needs.
\end{abstract}

It is interesting to note the comment made by Participant 5. This was one of the programmers coding APOSDLE not involved with the learning theory aspects of the system. He noticed the discussion was focusing on low-level design details while overlooking the personas' high-level goals i.e. focusing on how Learning Events were presented rather than asking whether they were useful in the first place given the personas' goals. This was an encouraging sign that some participants appreciated the personas' contribution and referred to them without the facilitators' intervention. As a result of a subsequent discussion it was decided to modify Learning Events in APOSDLE so they are presented as optional learning support an inexperienced employee like Paul might utilise, while not deterring more experienced employees from using the system. The next transcript illustrates how the personas were used to evaluate APOSDLE's collaboration component. It is used to formulate a collaboration request and share with the contacted person relevant resources before actual communication takes place using one of several means (e.g. phone, Skype, email).

Facilitator A: What kind of collaboration would Paul want to do?

Participant 7 (Programmer): If Paul has a visual basic problem, he won't hesitate to use the collaboration option. He will go there immediately and it will be very helpful if an expert with the relevant knowledge is suggested.

Facilitator B: Is he more likely to collaborate with an expert or another intern?

Participant 4 (Learning expert): He will turn to an intern first and then an expert, then a book and then an expert again

Participant 3 (Application Partner B): Interns have to prepare their question. He will ask his question according to some resources. He will look for something in the resource repository and if he doesn't understand something then he might consider a collaboration using APOSDLE Participant 8 (Application Partner C): Paul will first talk to his advisor.

Participant 9 (Knowledge management scientist): Will Paul like this kind of thing? I wonder if people will go through this process. I can imagine Paul simply pressing 'ask' and bypassing the form

Participant 10 (Programmer): What about a drag and drop? What if Paul finds a document but doesn't understand something he can drag it into the collaboration wizard and share it with the expert he is about to contact.

Facilitator B: Would Paul use the collaboration wizard to contact another fellow intern given there is no power distance between them?

Participant 3 (Application Partner B): [laugh] they would probably use Google Talk for that

Again, the discussion involved a number of participants from different professional backgrounds and the application partners contributed relevant contextual data. Given Paul's status as an intern it became clear that he might appreciate a structured collaboration request when contacting an expert in the company. However, when contacting someone at his own organisational level, he would either use an alternative more informal communication tool such as Google Talk or would opt to submit an unstructured request via APOSDLE, which will not require him to state his request in a rigid manner. As the sample transcripts show, getting participants to think about the system 
from the perspective of the personas helped articulate issues that we believe would have otherwise probably been overlooked. Referring to the personas helped focus discussions on end users and reduced the impact of personal preferences on the decision making process.

\section{Discussion}

In this section we discuss the strengths, weaknesses and limitations of using personas as part of our redesign efforts and specifically address the approach we adopted. As demonstrated by the discussion transcripts, when designing the $3^{\text {rd }}$ prototype of APOSDLE we focused solely on four fictitious individuals. We found that thinking about the system from the point of view of those people was by far more productive than referring to abstract terms such as User or Knowledge Seeker, which conveyed little if any meaningful data. We found personas a useful way of making information about end users, which tends to be scattered across several documents in different forms, visible so it could be easily referred to. It made such information tangible, meaningful and most importantly - referable by name. The personas were by no means seen as a perfect solution, however personifying key considerations such as the type of work users were involved in brought to the forefront essential consideration that probably would have been otherwise ignored or forgotten. Instructing participants to justify their suggestions by referring to a specific individual by name helped ensure the process was more user-centred and made people think explicitly about the individuals APOSDLE was meant to support.

In order for the personas to work, it was essential to ensure we were using the right personas i.e. realistic and valid representations of key user groups. Most of the accounts regarding persons we came across describe how personas were created based on methods such as interviews and observations. This raises questions concerning the validity of the end result and the way in which it is interpreted. Our collaborative approach sought to balance our own subjective interpretation of the data by involving stakeholders representing the intended end users in the creation and utilisation of the personas. Getting these stakeholders involved proved useful as they contributed data in the form of initial persona descriptions and validated the end result. Would we have been able to produce the personas without the application partners' contribution based solely on empirical data? We believe so, however we feel incorporating direct contributions from these stakeholders enriched the descriptions and made them more believable and ultimately more valid. This led to an effective buy-in process, which was essential in order for participants to accept and use the personas. We could have asked the application partners to merely review and comment on our personas but wanted them to feel a greater sense of ownership over the end result by getting involved.

The process of combining our own data with the application partners' contribution was not without its problems there are several limitations to the approach we have described in this paper. First, we relied on the application partners to provide vital data. At times this caused delays and in one case we did not receive the information we requested. The collaborative nature of the process meant our work developing the personas depended on the application partners, which was not ideal though ultimately beneficial in our view. Second, despite having provided a template, the style of the 
responses we received varied considerably. Consolidating the different styles in a consistent format across all four personas was challenging, but given the outcomes of the workshop we believe we achieved that goal successfully. In terms of utilising the personas in the workshop it was very helpful to have the application partners present. Because they were familiar and personally knew the people the personas represented, they helped interpret them. This eliminated ambiguity, uncertainty and disagreements among participants about the personas. However, as the design session progressed we observed that since the application partners' were seen as representatives of the personas, participants bypassed the personas by engaging with them directly. Not surprisingly, it was easier for them to ask a real person a question rather than attempt to view the system from someone else's perspective and make assumptions about a fictitious character. We view this as a weakness of our collaborative approach, which should be noted and addressed. Nevertheless, we do not think the application partners could have replaced the personas since each served a different purpose. The personas were first and foremost a communication tool, which personified user information and made it visible to project members thus enabling them to consider problems and solutions by thinking about the system from the point of view of different users. The stakeholders complemented and enriched the personas - they did not replace them. As facilitators, we felt at times that constantly reminding participants to refer to the personas to justify every design decision would have been potentially too annoying and counter-productive. This in our opinion is a significant weakness of the tool. Although we expected the personas to be 'present' at all time, we realise now this was not a very realistic expectation.

Despite the fact that during the workshop the personas were not referred to as rigorously as we had initially expected, they created a strong focus on APOSDLE's users and their goals, and as such we found them to be effective. Active participants appeared to be comfortable referring to Paul, Pierre, Eva and Lisa as they justified their ideas and assessed others' suggestions. For the most part, the persona-driven discussions resulted in actionable design decisions. We acknowledge that participants' familiarity with personas is essential to ensure they are naturally woven into the discussions. Overall, our experience using personas was a positive one and contributed significantly to our redesign efforts.

\section{Conclusions}

In this paper we described a case study of using personas to redesign the $3^{\text {rd }}$ prototype of the APOSDLE system. We used personas in order to explore how users' goals, behaviour and context could be communicated effectively to 21 project members during a two-day design workshop. The notion of designing with only a handful of specific individuals in mind has been debatable and some might argue - controversial. There are obvious pros and cons to the persona approach; however in our case we conclude the strengths outweighed the weaknesses. Keeping the end users in mind when considering design solutions is highly challenging and personal preferences, rather than the users' goals, can quickly end up dictating design decisions. We found personas to be an effective way to encapsulate and communicate user information so it served as constant reminder during discussions. Creating and utilising the personas was challenging yet beneficial. By combining initial persona descriptions generated by 
stakeholders with our empirical data we feel we were able to address to some degree methodological weaknesses and practical limitation of the tool expressed in the literature. The contribution of the user representatives helped in our view to increase the personas' validity and credibility, which were essential to ensure they were utilised successfully. Despite the fact the four personas were not referred to as rigorously as we had intended, we feel their contribution was nonetheless significant. They confronted project members with key questions regarding the ways APOSDLE could support the goals and needs of users in different industries and career stages. As reported by advocates of personas, we also maintain that personifying user information proved a useful way to communicate essential data about the end users, which despite possible inherent weaknesses was still better than any alternative we were familiar with.

Acknowledgments. APOSDLE (www.aposdle.org) is a 48 months integrated project partially funded by the European Community under the Information Society Technologies (IST) priority of the 6th Framework Programme for R\&D.

\section{References}

1. APOSDLE project internal deliverable - Formative Evaluation Report of 2nd Prototypes (2008)

2. Chapman, C.N., Milham, R.P.: The Persona's New Clothes: Methodological and Practical Arguments Against a Popular Method. In: Proceedings of the Human Factors and Ergonomics Society 50th Annual Meeting (2006)

3. Cooper, A.: The inmates are running the asylum. In: Why High-Tech Products drive us Crazy and How to Restore the Sanity, Macmillan, Indiana (1999)

4. Davis, F.D.: A Technology Acceptance Model for Empirically Testing New End-User Information Systems: Theory and Results. Doctoral dissertation, MIT Sloan School of Management, Cambridge MA (1986)

5. Gaver, W., Dunne, T., Pacenti, E.: Cultural probes. Interactions 6(1), 21-29 (1999)

6. Grudin, J., Pruitt, J.: Personas, Participatory Design and Product Development: An Infrastructure for Engagement. In: 7th Participatory Design Conference (PDC), pp. 144-161 (2002)

7. Kooken, J., Ley, T., de Hoog, R.: How Do People Learn at the Workplace? Investigating Four Workplace Learning Assumptions. In: Duval, E., Klamma, R., Wolpers, M. (eds.) EC-TEL 2007. LNCS, vol. 4753, pp. 158-171. Springer, Heidelberg (2007)

8. Lichtner, V., Kounkou, A.P., Dotan, A., Kooken, J.P., Maiden, N.M.A.: An Online Forum as User Diary for Remote Workplace Evaluation of a Work-Integrated Learning System. In: 27th Computer-Human Interaction Conference (in press, 2009)

9. Nieters, J.E., Ivaturi, S., Ahmed, I.: Making Personas Memorable. In: Conference on Human Factors in Computing Systems (CHI), pp. 1817-1824 (2007)

10. Pruitt, J., Adlin, T.: The Persona lifecycle. In: Keeping People in Mind Throughout Product Design. Morgan Kaufmann Publisher, San Francisco (2006)

11. Pruitt, J., Grudin, J.: Personas: practice and theory. In: Conference on Designing for User Experiences DUX 2003, ACM press, San Francisco (2003)

12. Sharp, H., Rogers, Y., Preece, J.: Interaction Design: Beyond Human-Computer Interaction, 2nd edn. Wiley, New-Jersey (2007) 